Pediatric Pulmonology

WILEY

\title{
EXHALED AIR TEMPERATURE IN CHILDREN WITH BRONCHOPULMONARY DYSPLASIA
}

\begin{tabular}{|c|c|}
\hline Journal: & Pediatric Pulmonology \\
\hline Manuscript ID: & PPUL-10-0007.R1 \\
\hline Wiley - Manuscript type: & Original Article \\
\hline $\begin{array}{r}\text { Date Submitted by the } \\
\text { Author: }\end{array}$ & 29-Apr-2010 \\
\hline Complete List of Authors: & $\begin{array}{l}\text { Carraro, Silvia; University of Padova, Department of Pediatrics } \\
\text { Piacentini, Giorgio; University of Verona, Department of Pediatrics } \\
\text { Lusiani, Marta; University of Padova, Department of Pediatrics } \\
\text { Uyan, Zeynep; University of Padova, Department of Pediatrics } \\
\text { Filippone, Marco; University of Padova, Department of Pediatrics } \\
\text { Schiavon, Maurizio; ULSS 16, Center for Sports Medicine and } \\
\text { Physical Activity, Department of Social Health } \\
\text { Boner, Attilio; University of Verona, Department of Pediatrics } \\
\text { Baraldi, Eugenio; University of Padova, Pediatrics }\end{array}$ \\
\hline Keywords: & $\begin{array}{l}\text { bronchopulmonary dysplasia, asthma, exhaled air temperature, } \\
\text { biomarkers }\end{array}$ \\
\hline
\end{tabular}

\section{scholarONE" \\ Manuscript Central}




\title{
EXHALED AIR TEMPERATURE IN CHILDREN WITH BRONCHOPULMONARY DYSPLASIA
}

\begin{abstract}
Silvia Carraro ${ }^{1,2}, \mathrm{MD}$, Giorgio Piacentini ${ }^{1,3}, \mathrm{MD}$, Marta Lusiani ${ }^{2}, \mathrm{MD}$, Zeynep S Uyan ${ }^{2}, \mathrm{MD}$, Marco Filippone ${ }^{2}, \mathrm{MD}$, Maurizio Schiavon ${ }^{4}, \mathrm{MD}$, Attilio L Boner ${ }^{3}, \mathrm{MD}$, Eugenio Baraldi ${ }^{2}, \mathrm{MD}$.
\end{abstract}

${ }^{1}$ These authors contributed equally to the article

${ }^{2}$ Deptartment of Pediatrics, University of Padova, Padova, Italy

${ }^{3}$ Deptartment of Pediatrics, University of Verona, Verona, Italy.

${ }^{4}$ Center for Sports Medicine and Physical Activity, Department of Social Health, ULSS 16, Padova, Italy

\section{Corresponding author}

Eugenio Baraldi, MD

Department of Pediatrics

Respiratory Medicine and Allergy Unit

University of Padova

Via Giustiniani 3

35128 Padova, Italy

Phone: +390498213560

Fax: $\quad+390498213502$

E-mail: baraldi@pediatria.unipd.it

Institution where the study was performed: Department of Pediatrics, University of Padova

Financial support: The work had no financial support

Running title: Exhaled air temperature in BPD 


\begin{abstract}
Background. Because they have similar functional and clinical profiles, bronchopulmonary dysplasia (BPD) survivors are often treated as asthmatic patients. In truth, very little is known about the possible biochemical and inflammatory mechanisms playing a part in BPD survivors' lungs. The aim of this study was to measure exhaled breath temperature in BPD survivors by comparison with asthmatic cases and healthy controls.
\end{abstract}

Methods. Three groups of age-matched adolescents $(n=17$ each), i.e. BPD survivors (gestational ages < 31 weeks, birth weights $<1500 \mathrm{~g}$ ), asthmatic subjects and healthy controls, underwent exhaled breath temperature and exhaled nitric oxide measurements, and spirometry.

Results. Exhaled breath temperature was significantly lower in the BPD survivors $\left(26.72^{\circ} \mathrm{C}[25.11-27.57]\right)$ than in the asthmatic patients $\left(29.60^{\circ} \mathrm{C}\right.$ [29.20-30.02], $\left.\mathrm{p}<0.001\right)$, while no significant difference emerged by comparison with healthy controls $\left(26.97^{\circ} \mathrm{C}\right.$ [26.58-27.38]). Considering the whole study population, a significant correlation was found between exhaled breath temperatures and exhaled nitric oxide concentrations $(R=0.42$ $\mathrm{p}=0.004)$. Spirometry revealed an obstructive lung function pattern in both the asthmatic cases and the BPD survivors, with lower parameters in the latter.

Conclusions. Exhaled breath temperatures and exhaled nitric oxide concentrations are significantly lower in BPD survivors than in asthmatic cases, suggesting that different pathogenetic mechanisms characterize these two chronic obstructive lung diseases.

Keywords: bronchopulmonary dysplasia; asthma; exhaled air temperature; biomarkers 


\section{INTRODUCTION}

Bronchopulmonary dysplasia (BPD) is the most common form of chronic lung disease of prematurity. In childhood and adolescence, BPD survivors may have respiratory symptoms and lung function impairment that closely resemble those of asthmatic patients, ${ }^{1}$ even if BPD patients present fewer acute exacerbations, poorer response to inhaled corticosteroids and less pronounced airway reversibility. A recent study reported that lowbirth-weight survivors have significantly higher odds of hospitalization for respiratory diseases (wheezing, respiratory infections and respiratory failure) as young adults than their normal-birth-weight counterparts. ${ }^{2}$

Although there are no data to suggest an asthma-like inflammatory profile in the airways of BPD survivors, they are often empirically treated as if they were asthmatic (e.g. with glucocorticosteroids). ${ }^{3,4}$ Very little is known, however, about the possible biochemical and inflammatory mechanisms involved in the pathogenesis of the symptoms and airway obstruction in BPD survivors, ${ }^{3}$ and no data are available on the pathological features of these patients' lungs beyond infancy. ${ }^{3}$

It is common knowledge that asthma is a chronic inflammatory disease of the airways and several non-invasive biomarkers have been proposed for indirectly assessing different patterns of airway inflammation. ${ }^{5}$ Exhaled nitric oxide has been studied the most and it is considered a reliable marker of eosinophilic inflammation in the airways. ${ }^{6,7}$ Since "calor" is one of the cardinal signs of inflammation, some authors recently recommended considering exhaled air temperature as a potential marker of inflammation. ${ }^{8-9}$ Increased values of exhaled breath temperature have been reported in children and adults with asthma ${ }^{8-11}$ correlating significantly with the concentrations of exhaled nitric oxide. ${ }^{8-10}$ To our knowledge, no studies have evaluated exhaled air temperature in individuals with BPD. 
The aim of the present cross-sectional study was to evaluate exhaled breath temperature (PLET, end-expiratory maneuver plateau temperature) in BPD survivors and to compare it with the temperature measured in age-matched asthmatic subjects and healthy controls.

\section{MATERIALS AND METHODS}

\section{Study subjects}

BPD survivors: 17 BPD survivors were considered (table), drawn from a population with BPD born at gestational ages below 31 weeks and with birth weights under $1500 \mathrm{~g}$ admitted to the Neonatal Intensive Care Unit between 1990 and 1994 and followed up routinely thereafter at the Pediatrics Department in Padova. BPD was defined as oxygen dependence persisting 28 days after birth in infants on mechanical ventilation (18 days on average). At birth, the mean gestational age of the sample was 28.3 weeks (SD 1.9) and the mean weight was $1011 \mathrm{~g}$ (SD 222).

All the BPD survivors were clinically stable at the time of recruitment, 5 of them were on low- to medium-dose inhaled steroid (ICS) treatment and two were being treated with theophylline. Two of the BPD survivors were sensitive to common airborne allergens (dust mites, dog and cat dander, grass and tree pollens).

Asthmatic subjects: 17 allergic asthmatic adolescents (table) who had been born at term were enrolled from among those regularly attending our outpatients clinic. Their asthma had been diagnosed by a pediatric respiratory physician based on their clinical history (cough, shortness of breath, recurrent wheezing, chest tightness) and an increase in FEV 1 after salbutamol $(400 \mu \mathrm{g})>12 \%$, according to international guidelines. ${ }^{12}$

All the asthmatic patients were clinically stable at the time of recruitment, 6 of them were on low- to medium-dose inhaled steroid (ICS) treatment $(100-200 \mathrm{mcg} /$ day budesonide equivalent) and one of them was being treated with long-acting beta2 agonists too. 
Healthy controls: 17 age-matched healthy adolescents (table) with no history of pre-term birth, atopy or respiratory diseases were recruited as a control group.

For all three groups, subjects who had suffered any airway infections in the previous 4 weeks were excluded from the study.

The Ethics Committee of our hospital reviewed and approved the protocol and all parents gave their informed consent.

At recruitment, all the adolescents underwent physical examination, exhaled air temperature measurement, $\mathrm{FE}_{\mathrm{NO}}$ measurement and spirometry. All the measurements were performed in the afternoon. $\mathrm{FE}_{\mathrm{NO}}$ measurement was not possible in 3 subjects (1 with asthma and 2 with BPD)

\section{Exhaled breath temperature (PLET)}

Exhaled breath temperature (end-expiratory maneuver plateau temperature, PLET) was measured as described elsewhere. ${ }^{13}$ The environmental conditions were controlled in order to maintain a stable temperature of $23 \pm 1^{\circ} \mathrm{C}$ and a relative humidity of $40-50 \%$.

The measurements were repeated three times. The inspiratory air stream temperature was recorded during a complete inhalation to total lung capacity (TLC). The exhaled airway temperature was measured during a slow exhalation to residual volume (RV) using the Medical Mass Flow Sensor of the Vmax Spectra 229 Pulmonary Function Laboratory (VIASYS Healthcare, Yorba Linda, CA, USA). Dynamic airway temperature and airflow were measured with the Mass Flow Sensor. The response time of the Mass Flow Sensor to a steep change in airway temperature was approximately 64 milliseconds, with a thermal resolution of $0.01^{\circ} \mathrm{C}$. Mouth pressure was measured at the airway opening using a differential pressure transducer. Exhaled flow was directed through an alternate pathway with a fixed resistor in place to control the expiratory air flow rate and maintain an airway pressure sufficient to keep the velum closed and prevent any contribution of warmed gas from nasal sinuses. A mouth pressure of $>5 \mathrm{~cm} \mathrm{H}$ O also helped the subject to stabilize 
the expiratory flow at 5-6 I/min. All the signals, including exhaled air temperature, mouth pressure and exhalation flow rate, were transferred to a personal computer and analyzed using software specifically developed for this purpose (Vmax Temperature, VIASYS Healthcare, Yorba Linda, CA, USA). Real-time signal display consisted of inspiratory and expiratory airway temperature, respiratory flow rate and mouth (airway) pressure on a time axis. Flow and pressure limit indicators were presented on screen as a coaching aid.

At the end of the test, the software automatically calculated the PLET, which was the parameter used to compare the groups.

\section{Fractional exhaled nitric oxide ( $\left.F E_{\mathrm{NO}}\right)$ and pulmonary function measurement}

$\mathrm{FE}_{\mathrm{NO}}$ was measured using the NIOX system (Aerocrine, Stockholm, Sweden), according to the ERS/ATS guidelines for measuring $\mathrm{FE}_{\mathrm{NO}}$ in children. ${ }^{14}$ Children inhaled NO-free air to total lung capacity (TLC) and exhaled through a dynamic flow restrictor with a target flow of $50 \mathrm{ml} / \mathrm{sec}$ for at least 6-7 seconds. No nose clip was used. The NIOX system was calibrated using a 200 ppb NO tank (Lindegas Hoek Loos Speciality Gases, Amsterdam, Netherlands) according to the manufacturer's instructions.

Lung function was measured by means of a 10-liter bell spirometer (Biomedin, Padova, Italy) and the best of three maneuvers was expressed as a percentage (\%) of the predicted reference values, according to the ERS/ATS guidelines. ${ }^{15}$

\section{Statistical analysis}

It has been calculated that a sample size of 17 subjects allows detecting a $1^{\circ} \mathrm{C}$ difference between groups of with a power of $80 \%$ at a 2 -sided $\square$-level of .05 .

Age and anthropometric data were expressed as the mean and standard deviation (SD) (table).

Since PLET, FE $_{\mathrm{NO}}$ and lung function data were not normally distributed (as demonstrated by the Shapiro-Wilk test), they were expressed as medians and interquartile ranges (IQR), 
and analyzed by means of non-parametric tests. Differences between groups were analyzed using the Kruskal-Wallis test, followed (where significant) by the Mann-Whitney U-test for comparisons between groups. Correlations were evaluated using Spearman's rank test. Statistical significance was set at a 2 -sided alpha level of 0.05 .

\section{RESULTS}

\section{Exhaled breath temperature (PLET)}

The Kruskal Wallis test revealed a significant difference in the PLET for the 3 groups $(p<0.001)$ (figure 1A). The between-group comparison found a significantly lower PLET in BPD survivors $\left(26.72^{\circ} \mathrm{C}\right.$ [25.11-27.57]) than in asthmatic patients $\left(29.60^{\circ} \mathrm{C}\right.$ [29.20-30.02], $p<0.001$ ), while no significant difference emerged between BPD survivors and healthy controls $\left(26.97^{\circ} \mathrm{C}[26.58-27.38] \mathrm{p}=0.23\right)$. The asthmatic cases had a significantly higher PLET than the healthy controls $(p<0.001)$.

\section{Exhaled nitric oxide (FE $\mathrm{FO}_{\mathrm{NO}}$}

The Kruskal Wallis test found a significant difference in $\mathrm{FE}_{\mathrm{NO}}$ for the 3 groups $(p=0.003)$ (figure $1 \mathrm{~B}$ ). The between-group comparison revealed a significantly higher $\mathrm{FE}_{\mathrm{NO}}$ in asthmatic cases (29.9 ppb [15.0-49.2]) than in either healthy controls (11.7 ppb [8.5-16.8], $\mathrm{p}=0.008$ ) or BPD survivors (10.6 ppb [7.0-13.2], $\mathrm{p}=0.002)$, with no significant difference between the latter two groups $(p=0.28)$.

\section{Lung function}

The Kruskal Wallis test disclosed significant differences in all the spirometric parameters. The between-group comparison showed that BPD children had lower FVC, FEV $1, F F_{25-75}$ and $\mathrm{FEV}_{1} / \mathrm{FVC}$ than either asthmatic cases or healthy controls; and the asthmatic cases had lower $\mathrm{FEV}_{1}, \mathrm{FEF}_{25-75}$ and $\mathrm{FEV}_{1} / \mathrm{FVC}$ than healthy controls (table, figure $1 \mathrm{C}$ and $1 \mathrm{D}$ ). 


\section{Correlations}

Considering the whole sample, a significant correlation emerged between PLET and FE $\mathrm{NO}_{\mathrm{NO}}$ $(R=0.42 p=0.004)$ (figure 2).

In BPD survivors there was a correlation approaching statistical significance between FVC and PLET ( $p=0.058 R=0.47)$, which was stronger considering the whole sample $(R=0.28$ $\mathrm{p}=0.036)$

None of the other spirometric parameters correlated with PLET values in BPD survivors. No significant correlations emerged between spirometric measurements and PLET in asthmatic cases or healthy controls.

\section{DISCUSSION}

This study shows that exhaled breath temperature is significantly lower in BPD survivors than in asthmatic patients.

BPD survivors have respiratory symptoms and lung function profiles that resemble those of asthmatic children ${ }^{16}$ and this explains why they are often considered and empirically treated as asthmatic patients during their childhood. ${ }^{3}$ Very little it is known about the processes underlying the obstructive lung function pattern in BPD survivors and there is no evidence to support any asthma-like inflammation in this condition. ${ }^{4,17,18}$

Here we demonstrate that both PLET and $\mathrm{FE}_{\mathrm{NO}}$ are lower in BPD survivors than in asthmatic children, suggesting that the two conditions have a different pathogenesis (figure $1 \mathrm{~A}$ and $1 \mathrm{~B}$ ). In a previous study, we already showed that exhaled NO is lower in BPD survivors than in asthmatic cases; ${ }^{19}$ the present study further supports the lack of any asthma-like airway inflammation in BPD survivors. A possible limit in the interpretation of our results is the potential effect of ICS treatment and atopy on PLET values. In fact while all asthmatic patients were allergic, only two BPD patients were sensitized. In addition some patients in both groups were treated with ICS. 
In keeping with previous studies conducted on asthmatic patients, ${ }^{8-9}$ PLET and FE $_{\mathrm{NO}}$ correlated significantly. It is worth noting that the scatter plot (figure 2) shows a clear separation between asthmatic cases and BPD survivors, again suggesting that different pathogenetic mechanisms underlie these two chronic obstructive lung diseases.

As previously reported, ${ }^{8-10}$ asthmatic subjects have higher levels of both FE $_{\mathrm{NO}}$ and PLET, which are thought to reflect eosinophilic airway inflammation. ${ }^{6,20}$ The higher exhaled breath temperature in asthmatic subjects also seems to suggest an increased vascularity and bronchial blood flow. ${ }^{10}$ In BPD survivors, on the other hand, PLET and FE $\mathrm{NO}_{\mathrm{NO}}$ are significantly lower than in asthmatic patients and even tend to be lower than in healthy controls, although without reaching the statistical significance (figure $1 \mathrm{~A}$ and $1 \mathrm{~B}$ ). This trend could be explained by the anatomical alterations that characterize BPD lungs, namely alveolar simplification, variable degree of interstitial fibrosis ${ }^{21,22}$ and dysmorphic vascular growth. ${ }^{23}$ These anatomical alterations might presumably be responsible for an impaired heat exchange between the bronchial vessels and the airway lumen, giving rise to normal-to-low values of exhaled air temperature in BPD survivors.

As previously reported, ${ }^{24}$ we confirm here that BPD survivors have a lung function pattern with airflow limitation (figure 1C). An autoptic study demonstrated thickening of the airway walls and an increase in smooth muscle, suggesting that these aspects may have a role in airway obstruction and hyper-responsiveness in BPD survivors. ${ }^{25}$ Moreover BPD survivors have a significantly reduced forced vital capacity (figure 1D). This last features might be explained by the impaired growth of the BPD survivor's lungs, which would lead to reduced pulmonary volumes. Previous studies have already documented in BPD survivors an impaired lung function. ${ }^{24,26,27}$ It may be that lung function impairment in BPD survivors is not just the static outcome of the initial damage, but due to an ongoing remodeling/damaging process. Although our findings go against any suggestion of an asthma-like eosinophilic inflammatory profile, other inflammatory pathways (e.g. 
neutrophilic or lymphocytic) and/or remodeling mechanisms may be involved. Elucidating this pathophysiological substrate could have an impact on the long-term management of BPD subjects.

In conclusion, we showed that exhaled breath temperature (PLET) and exhaled nitric oxide (which are considered biomarkers of airway inflammation) are not increased in BPD survivors. Further studies are needed to assess the role of other non-invasive biomarkers, as measured for instance in exhaled breath condensate, to shed light on the pathogenic processes affecting the BPD survivor's lung and thus pave the way to the development of new, specifically targeted drugs.

\section{Acknowledgement}

The authors thank VIASYS Healthcare, Yorba Linda, CA, USA, for providing hardware and software for exhaled air evaluation. 


\section{REFERENCES}

1. Greenough A. Bronchopulmonary dysplasia--long term follow up. Paediatr Respir Rev 2006;7:S189-91.

2. Walter EC, Ehlenbach WJ, Hotchkin DL, Chien JW, Koepsell TD. Low Birthweight and Respiratory Disease in Adulthood: A Population-Based Case-Control Study. Am J Respir Crit Care Med 2009;180:176-80

3. Baraldi E, Filippone M. Chronic lung disease after premature birth. N Engl J Med 2007;357:1946-1955.

4. Narang I, Baraldi E, Silverman M, Bush A. Airway function measurements and the long-term follow-up of survivors of preterm birth with and without chronic lung disease. Pediatr Pulmonol 2006;41:497-508.

5. Kharitonov SA, Barnes PJ. Exhaled markers of pulmonary disease. Am J Respir Crit Care Med 2001;163:1693-1722.

6. Berry MA, Shaw DE, Green RH, Brightling CE, Wardlaw AJ, Pavord ID. The use of exhaled nitric oxide concentration to identify eosinophilic airway inflammation: an observational study in adults with asthma. Clin Exp Allergy 2005;35:1175-1179.

7. Baraldi E, Azzolin NM, Zanconato S, Dario C, Zacchello F. Corticosteroids decrease exhaled nitric oxide in children with acute asthma. $J$ Pediatr $1997 ; 131: 381-385$

8. Piacentini GL, Bodini A, Zerman L, Costella S, Zanolla L, Peroni DG, Boner AL. Relationship between exhaled air temperature and exhaled nitric oxide in childhood asthma. Eur Respir J $2002 ; 20: 108-111$.

9. Paredi P, Kharitonov SA, Barnes PJ. Faster rise of exhaled breath temperature in asthma: a novel marker of airway inflammation? Am J Respir Crit Care Med $2002 ; 165: 181-184$ 
10. Paredi P, Kharitonov SA, Barnes PJ. Correlation of exhaled breath temperature with bronchial blood flow in asthma. Respir Res 2005 ;6:15.

11. Pifferi M, Ragazzo V, Previti A, Pioggia G, Ferro M, Macchia P, Piacentini GL, Boner AL. Exhaled air temperature in asthmatic children: a mathematical evaluation. Pediatr Allergy Immunol 2009;20:164-171.

12. Global Strategy for Asthma Management and Prevention, Global Initiative for Asthma (GINA) 2008. Available from: http://www.ginasthma.org.

13. Piacentini GL, Peroni D, Crestani E, Zardini F, Bodini A, Costella S, Boner AL. Exhaled air temperature in asthma: methods and relationship with markers of disease. Clin Exp Allergy 2007;37:415-419

14. Baraldi E, de Jongste JC; European Respiratory Society; American Thoracic Society. Measurement of exhaled nitric oxide in children, 2001. Eur Respir J 2002;20:223-237.

15. Miller MR, Hankinson J, Brusasco V, et al; ATS/ERS Task Force. Standardisation of spirometry. Eur Respir J 2005 ; 26:319-338.

16. Vrijlandt EJ, Boezen HM, Gerritsen J, Stremmelaar EF, Duiverman EJ. Respiratory health in prematurely born preschool children with and without bronchopulmonary dysplasia. J Pediatr 2007;150:256-261.

17. Allen J, Zwerdling R, Ehrenkranz R, et al. Statement on the care of the child with chronic lung disease of infancy and childhood. Am $\mathrm{J}$ Respir Crit Care Med 2003;168:356-396

18. Bhandari A, Bhandari V. Pitfalls, problems, and progress in bronchopulmonary dysplasia. Pediatrics 2009;123:1562-1573

19. Baraldi E, Bonetto G, Zacchello F, Filippone M. Low exhaled nitric oxide in schoolage children with bronchopulmonary dysplasia and airflow limitation. Am J Respir Crit Care Med 2005;171:68-72. 
20.Piacentini GL, Bodini A, Peroni D, Ress M, Costella S, Boner AL. Exhaled air temperature and eosinophil airway inflammation in allergic asthmatic children. $\mathrm{J}$ Allergy Clin Immunol 2004;114:202-204.

21. Thébaud B, Abman SH. Bronchopulmonary dysplasia: where have all the vessels gone? Roles of angiogenic growth factors in chronic lung disease. Am J Respir Crit Care Med 2007;175:978-985.

22. Coalson JJ. Pathology of bronchopulmonary dysplasia. Semin Perinatol 2006;30:179-184.

23. Abman $\mathrm{SH}$. The dysmorphic pulmonary circulation in bronchopulmonary dysplasia: a growing story. Am J Respir Crit Care Med 2008;178:114-115

24. Filippone M, Sartor M, Zacchello F, Baraldi E. Flow limitation in infants with bronchopulmonary dysplasia and respiratory function at school age. Lancet 2003;361:753-754.

25. Tiddens HA, Hofhuis W, Casotti V, Hop WC, Hulsmann AR, de Jongste JC. Airway dimensions in bronchopulmonary dysplasia: implications for airflow obstruction. Pediatr Pulmonol 2008;43:1206-13.

26. Jacob SV, Coates AL, Lands LC, MacNeish CF, Riley SP, Hornby L, Outerbridge EW, Davis GM, Williams RL. Long-term pulmonary sequelae of severe bronchopulmonary dysplasia. J Pediatr 1998;133:193-200.

27. Gross SJ, lannuzzi DM, Kveselis DA, Anbar RD. Effect of preterm birth on pulmonary function at school age: a prospective controlled study. J Pediatr 1998;133:188-192. 


\section{FIGURE LEGENDS}

Figure 1. Panel A shows PLET values in the 3 groups; panel $B$ shows $F E_{N O}$ values in the 3 groups; panel $C$ shows FVC values in the 3 groups; panel D shows FEV1 values in the 3 groups. ${ }^{* *} p<0.001$ and ${ }^{*} p<0.01$ vs healthy controls; $\# p<0.05$ and $\$ p<0.01$ vs asthmatic subjects; $\gamma \quad p<0.001$ vs BPD survivors

Figure 2. Correlation between $\mathrm{FE}_{\mathrm{NO}}$ values and PLET values in BPD, asthmatic and healthy subjects $(R=0.42 \mathrm{p}=0.004)$. 


\begin{abstract}
Silvia Carraro ${ }^{1,2}, \mathrm{MD}$, Giorgio Piacentini ${ }^{1,3}, \mathrm{MD}$, Marta Lusiani ${ }^{2}, \mathrm{MD}$, Zeynep S Uyan ${ }^{2}, \mathrm{MD}$, Marco Filippone ${ }^{2}, \mathrm{MD}$, Maurizio Schiavon ${ }^{4}, \mathrm{MD}$, Attilio L Boner ${ }^{3}, \mathrm{MD}$, Eugenio Baraldi ${ }^{2}, \mathrm{MD}$.
\end{abstract}

\title{
EXHALED AIR TEMPERATURE IN CHILDREN WITH BRONCHOPULMONARY DYSPLASIA
}

\footnotetext{
${ }^{1}$ These authors contributed equally to the article

${ }^{2}$ Deptartment of Pediatrics, University of Padova, Padova, Italy

${ }^{3}$ Deptartment of Pediatrics, University of Verona, Verona, Italy.

${ }^{4}$ Center for Sports Medicine and Physical Activity, Department of Social Health, ULSS 16, Padova, Italy
}

\section{Corresponding author}

Eugenio Baraldi, MD Department of Pediatrics

Respiratory Medicine and Allergy Unit University of Padova

Via Giustiniani 3 35128 Padova, Italy

Phone: +390498213560

Fax: $\quad+390498213502$

E-mail: baraldi@pediatria.unipd.it

Institution where the study was performed: Department of Pediatrics, University of Padova

Financial support: The work had no financial support

Running title: Exhaled air temperature in BPD 


\begin{abstract}
Background. Because they have similar functional and clinical profiles, bronchopulmonary dysplasia (BPD) survivors are often treated as asthmatic patients. In truth, very little is known about the possible biochemical and inflammatory mechanisms playing a part in BPD survivors' lungs. The aim of this study was to measure exhaled breath temperature (an indirect marker of airway inflammation) in BPD survivors by comparison with asthmatic cases and healthy controls.

Methods. Three groups of age-matched adolescents ( $n=17$ each), i.e. BPD survivors (gestational ages < 31 weeks, birth weights < $1500 \mathrm{~g}$ ), asthmatic subjects and healthy controls, underwent exhaled breath temperature and exhaled nitric oxide measurements, and spirometry.
\end{abstract}

Results. Exhaled breath temperature was significantly lower in the BPD survivors $\left(26.72^{\circ} \mathrm{C}[25.11-27.57]\right)$ than in the asthmatic patients $\left(29.60^{\circ} \mathrm{C}\right.$ [29.20-30.02], $\left.\mathrm{p}<0.001\right)$, while no significant difference emerged by comparison with healthy controls $\left(26.97^{\circ} \mathrm{C}\right.$ [26.58-27.38]). Considering the whole study population, a significant correlation was found between exhaled breath temperatures and exhaled nitric oxide concentrations ( $R=0.42$ $\mathrm{p}=0.004)$. Spirometry revealed an obstructive lung function pattern in both the asthmatic cases and the BPD survivors, with lower parameters in the latter.

Conclusions. Exhaled breath temperatures and exhaled nitric oxide concentrations are significantly lower in BPD survivors than in asthmatic cases, suggesting that different pathogenetic mechanisms characterize these two chronic obstructive lung diseases.

Keywords: bronchopulmonary dysplasia; asthma; exhaled air temperature; biomarkers 


\section{INTRODUCTION}

Bronchopulmonary dysplasia (BPD) is the most common form of chronic lung disease of prematurity. In childhood and adolescence, BPD survivors may have respiratory symptoms and lung function impairment that closely resemble those of asthmatic patients, ${ }^{1}$ even if BPD patients present fewer acute exacerbations, poorer response to inhaled corticosteroids and less pronounced airway reversibility hough they do not share the same-origins. A recent study reported that low-birth-weight survivors have significantly higher odds of hospitalization for respiratory diseases (wheezing, respiratory infections and respiratory failure) as young adults than their normal-birth-weight counterparts. ${ }^{2}$

Although there are no data to suggest an asthma-like inflammatory profile in the airways of BPD survivors, they are often empirically treated as if they were asthmatic (e.g. with glucocorticosteroids). ${ }^{3,4}$ Very little is known, however, about the possible biochemical and inflammatory mechanisms involved in the pathogenesis of the symptoms and airway obstruction in BPD survivors, ${ }^{3}$ and no data are available on the pathological features of these patients' lungs beyond infancy. ${ }^{3}$

It is common knowledge that asthma is a chronic inflammatory disease of the airways and several non-invasive biomarkers have been proposed for indirectly assessing different patterns of airway inflammation. ${ }^{5}$ Exhaled nitric oxide has been studied the most and it is considered a reliable marker of eosinophilic inflammation in the airways. ${ }^{6,7}$ Since "calor" is one of the cardinal signs of inflammation, some authors recently recommended considering exhaled air temperature as a potential marker of inflammation. ${ }^{8-9}$ Increased values of exhaled breath temperature have been reported in children and adults with asthma ${ }^{8-11}$ correlating significantly with the concentrations of exhaled nitric oxide. ${ }^{8-10}$ To our knowledge, no studies have evaluated exhaled air temperature in individuals with BPD. 
The aim of the present cross-sectional study was to evaluate exhaled breath temperature (PLET, end-expiratory maneuver plateau temperature) in BPD survivors and to compare it with the temperature measured in age-matched asthmatic subjects and healthy controls.

\section{MATERIALS AND METHODS}

\section{Study subjects}

BPD survivors: 17 BPD survivors were considered (table), drawn from a population with BPD born at gestational ages below 31 weeks and with birth weights under $1500 \mathrm{~g}$ admitted to the Neonatal Intensive Care Unit between 1990 and 1994 and followed up routinely thereafter at the Pediatrics Department in Padova. BPD was defined as oxygen dependence persisting 28 days after birth in infants on mechanical ventilation (18 days on average). At birth, the mean gestational age of the sample was 28.3 weeks (SD 1.9) and the mean weight was $1011 \mathrm{~g}$ (SD 222).

All the BPD survivors were clinically stable at the time of recruitment, 5 of them were on low- to medium-dose inhaled steroid treatment and two were being treated with theophylline. Two of the BPD survivors were sensitive to common airborne allergens (dust mites, dog and cat dander, grass and tree pollens).

Asthmatic subjects: 17 allergic asthmatic adolescents (table) who had been born at term were enrolled from among those regularly attending our outpatients clinic. Their asthma had been diagnosed by a pediatric respiratory physician based on their clinical history (cough, shortness of breath, recurrent wheezing, chest tightness) and an increase in FEV 1 after salbutamol $(400 \mu \mathrm{g})>12 \%$, according to international guidelines. ${ }^{12}$

All the asthmatic patients were clinically stable at the time of recruitment, 6 of them were on low- to medium-dose inhaled steroid treatment (100-200 mcg/day budesonide equivalent) and one of them was being treated with long-acting beta2 agonists too. 
Healthy controls: 17 age-matched healthy adolescents (table) with no history of pre-term birth, atopy or respiratory diseases were recruited as a control group.

For all three groups, subjects who had suffered any airway infections in the previous 4 weeks were excluded from the study.

The Ethics Committee of our hospital reviewed and approved the protocol and all parents gave their informed consent.

At recruitment, all the adolescents underwent physical examination, exhaled air temperature measurement, $\mathrm{FE}_{\mathrm{NO}}$ measurement and spirometry. All the measurements were performed in the afternoon. $\mathrm{FE}_{\mathrm{NO}}$ measurement was not possible in 3 subjects (1 with asthma and 2 with BPD)

\section{Exhaled breath temperature (PLET)}

Exhaled breath temperature (end-expiratory maneuver plateau temperature, PLET) was measured as described elsewhere. ${ }^{13}$ The environmental conditions were controlled in order to maintain a stable temperature of $23 \pm 1^{\circ} \mathrm{C}$ and a relative humidity of $40-50 \%$.

The measurements were repeated three times. The inspiratory air stream temperature was recorded during a complete inhalation to total lung capacity (TLC). The exhaled airway temperature was measured during a slow exhalation to residual volume (RV) using the Medical Mass Flow Sensor of the Vmax Spectra 229 Pulmonary Function Laboratory (VIASYS Healthcare, Yorba Linda, CA, USA). Dynamic airway temperature and airflow were measured with the Mass Flow Sensor. The response time of the Mass Flow Sensor to a steep change in airway temperature was approximately 64 milliseconds, with a thermal resolution of $0.01^{\circ} \mathrm{C}$. Mouth pressure was measured at the airway opening using a differential pressure transducer. Exhaled flow was directed through an alternate pathway with a fixed resistor in place to control the expiratory air flow rate and maintain an airway pressure sufficient to keep the velum closed and prevent any contribution of warmed gas from nasal sinuses. A mouth pressure of $>5 \mathrm{~cm} \mathrm{H}_{2} \mathrm{O}$ also helped the subject to stabilize 
the expiratory flow at 5-6 I/min. All the signals, including exhaled air temperature, mouth pressure and exhalation flow rate, were transferred to a personal computer and analyzed using software specifically developed for this purpose (Vmax Temperature, VIASYS Healthcare, Yorba Linda, CA, USA). Real-time signal display consisted of inspiratory and expiratory airway temperature, respiratory flow rate and mouth (airway) pressure on a time axis. Flow and pressure limit indicators were presented on screen as a coaching aid.

At the end of the test, the software automatically calculated the PLET, which was the parameter used to compare the groups.

\section{Fractional exhaled nitric oxide $\left(\mathrm{FE}_{\mathrm{NO}}\right)$ and pulmonary function measurement}

$\mathrm{FE}_{\mathrm{NO}}$ was measured using the NIOX system (Aerocrine, Stockholm, Sweden), according to the ERS/ATS guidelines for measuring $\mathrm{FE}_{\mathrm{NO}}$ in children. ${ }^{14}$ Children inhaled NO-free air to total lung capacity (TLC) and exhaled through a dynamic flow restrictor with a target flow of $50 \mathrm{ml} / \mathrm{sec}$ for at least 6-7 seconds. No nose clip was used. The NIOX system was calibrated using a 200 ppb NO tank (Lindegas Hoek Loos Speciality Gases, Amsterdam, Netherlands) according to the manufacturer's instructions.

Lung function was measured by means of a 10-liter bell spirometer (Biomedin, Padova, Italy) and the best of three maneuvers was expressed as a percentage (\%) of the predicted reference values, according to the ERS/ATS guidelines. ${ }^{15}$

\section{Statistical analysis}

It has been calculated that a sample size of 17 subjects allows detecting a $1^{\circ} \mathrm{C}$ difference between groups of with a power of $80 \%$ at a 2 -sided $\square$-level of .05 .

Age and anthropometric data were expressed as the mean and standard deviation (SD) (table).

Since PLET, FE $_{\mathrm{NO}}$ and lung function data were not normally distributed (as demonstrated by the Shapiro-Wilk test), they were expressed as medians and interquartile ranges (IQR), 
and analyzed by means of non-parametric tests. Differences between groups were analyzed using the Kruskal-Wallis test, followed (where significant) by the Mann-Whitney U-test for comparisons between groups. Correlations were evaluated using Spearman's rank test. Statistical significance was set at a 2 -sided $\alpha$ level of 0.05 .

\section{RESULTS}

\section{Exhaled breath temperature (PLET)}

The Kruskal Wallis test revealed a significant difference in the PLET for the 3 groups $(p<0.001)$ (figure 1A). The between-group comparison found a significantly lower PLET in BPD survivors $\left(26.72^{\circ} \mathrm{C}\right.$ [25.11-27.57]) than in asthmatic patients $\left(29.60^{\circ} \mathrm{C}\right.$ [29.20-30.02], $p<0.001$ ), while no significant difference emerged between BPD survivors and healthy controls $\left(26.97^{\circ} \mathrm{C}[26.58-27.38] \mathrm{p}=0.23\right)$. The asthmatic cases had a significantly higher PLET than the healthy controls $(p<0.001)$.

\section{Exhaled nitric oxide (FE NO$)$}

The Kruskal Wallis test found a significant difference in $F E_{N O}$ for the 3 groups $(p=0.003)$ (figure $1 \mathrm{~B}$ ). The between-group comparison revealed a significantly higher $\mathrm{FE}_{\mathrm{NO}}$ in asthmatic cases (29.9 ppb [15.0-49.2]) than in either healthy controls (11.7 ppb [8.5-16.8], $\mathrm{p}=0.008$ ) or BPD survivors (10.6 ppb [7.0-13.2], $\mathrm{p}=0.002$ ), with no significant difference between the latter two groups $(p=0.28)$.

\section{Lung function}

The Kruskal Wallis test disclosed significant differences in all the spirometric parameters. The between-group comparison showed that BPD children had lower FVC, FEV 1, FEF $_{25-75}$ and $\mathrm{FEV}_{1} / \mathrm{FVC}$ than either asthmatic cases or healthy controls; and the asthmatic cases had lower $\mathrm{FEV}_{1}, \mathrm{FEF}_{25-75}$ and $\mathrm{FEV}_{1} / \mathrm{FVC}$ than healthy controls (table, figure $1 \mathrm{C}$ and $1 \mathrm{D}$ ). 


\section{Correlations}

Considering the whole sample, a significant correlation emerged between PLET and FE $\mathrm{NO}_{\mathrm{NO}}$ $(R=0.42 p=0.004)$ (figure 2).

In BPD survivors there was a correlation approaching statistical significance between FVC and PLET ( $p=0.058 R=0.47)$, which was stronger considering the whole sample ( $R=0.28$ $p=0.036$ hereas Nane of the other spirometric parameters correlated with PLET values in BPD survivors.

No significant correlations emerged between spirometric measurements and PLET in asthmatic cases or healthy controls.

\section{DISCUSSION}

This study shows that exhaled breath temperature is significantly lower in BPD survivors than in asthmatic patients.

BPD survivors have respiratory symptoms and lung function profiles that resemble those of asthmatic children ${ }^{16}$ and this explains why they are often considered and empirically treated as asthmatic patients during their childhood. ${ }^{3}$ Very little it is known about the processes underlying the obstructive lung function pattern in BPD survivors and there is no evidence to support any asthma-like inflammation in this condition. ${ }^{4,17,18}$

Here we demonstrate that both PLET and $\mathrm{FE}_{\mathrm{NO}}$ are lower in BPD survivors than in asthmatic children, strongly suggesting that the two conditions have a different pathogenesis (figure $1 \mathrm{~A}$ and $1 \mathrm{~B}$ ). In a previous study, we already showed that exhaled NO is lower in BPD survivors than in asthmatic cases; ${ }^{19}$ the present study further supports the lack of any asthma-like airway inflammation in BPD survivors.

A possible limit in the interpretation of our results is the potential effect of ICS treatment and atopy on PLET values. In fact while all asthmatic patients were allergic, only two BPD patients were sensitized. In addition some patients in both groups were treated with ICS. 
In keeping with previous studies conducted on asthmatic patients, ${ }^{8-9}$ PLET and $\mathrm{FE}_{\mathrm{NO}}$ correlated significantly. It is worth noting that the scatter plot (figure 2) shows a clear separation between asthmatic cases and BPD survivors, again suggesting that different pathogenetic mechanisms underlie these two chronic obstructive lung diseases.

As previously reported, ${ }^{8-10}$ asthmatic subjects have higher levels of both $\mathrm{FE}_{\mathrm{NO}}$ and PLET, which are thought to reflect eosinophilic airway inflammation. ${ }^{6,20}$ The higher exhaled breath temperature in asthmatic subjects also seems to suggest an increased vascularity and bronchial blood flow. ${ }^{10}$ In BPD survivors, on the other hand, PLET and $\mathrm{FE}_{\mathrm{NO}}$ are significantly lower than in asthmatic patients and even tend to be lower than in healthy controls, although without reaching the statistical significance (figure $1 \mathrm{~A}$ and $1 \mathrm{~B}$ ). This trend could be explained by the anatomical alterations that characterize BPD lungs, namely alveolar simplification, variable degree of interstitial fibrosis ${ }^{21,22}$ and dysmorphic vascular growth. ${ }^{23}$ These anatomical alterations might presumably be responsible for an impaired heat exchange between the bronchial vessels and the airway lumen, giving rise to normal-to-low values of exhaled air temperature in BPD survivors.

As previously reported, ${ }^{24}$ we confirm here that BPD survivors have a lung function pattern with airflow limitation (figure 1C). An autoptic study demonstrated thickening of the airway walls and an increase in smooth muscle, suggesting that these aspects may have a role in airway obstruction and hyper-responsiveness in BPD survivors. ${ }^{25}$ Moreover BPD survivors have a significantly reduced forced vital capacity (figure 1D). This last features might be explained by the impaired growth of the BPD survivor's lungs, which would lead to reduced pulmonary volumes.

Previous studies have already documented an impaired lung function in BPD survivors an impaired lung function and characterized by an accelerated decline. ${ }^{24,26,27}$ In a follow-up study, Doyle et at ${ }^{28}$ reported significant airflow obstruction in BPD survivors at 18 years of age, with an accelerated decline in their $F E V_{1} / F V C$ ratio, suggesting an earlier 
deterioration in their respiratory health than in the general population. Lung function declines physiologically in adulthood and senescence, albeit without reaching levels associated with disability. ${ }^{29}$ This decline could be more of a problem in BPD survivors, whose maximal lung function is underdeveloped. It may be that lung function impairment in BPD survivors is not just the static outcome of the initial damage, but due to an ongoing remodeling/damaging process. Although our findings go against any suggestion of an asthma-like eosinophilic inflammatory profile, other inflammatory pathways (e.g. neutrophilic or lymphocytic) and/or remodeling mechanisms may be involved. Elucidating this pathophysiological substrate could have a great impact on the long-term management of BPD subjects.

In conclusion, we showed that exhaled breath temperature (PLET) and exhaled nitric oxide (which are considered biomarkers of eosinophilie airway inflammation) are not increased in BPD survivors. Further studies are needed to assess the role of other noninvasive biomarkers, as measured for instance in exhaled breath condensate, to shed light on the pathogenic processes affecting the BPD survivor's lung and thus pave the way to the development of new, specifically targeted drugs.

\section{Acknowledgement}

The authors thank VIASYS Healthcare, Yorba Linda, CA, USA, for providing hardware and software for exhaled air evaluation. 


\section{REFERENCES}

1. Greenough A. Bronchopulmonary dysplasia--long term follow up. Paediatr Respir Rev 2006;7:S189-91.

2. Walter EC, Ehlenbach WJ, Hotchkin DL, Chien JW, Koepsell TD. Low Birthweight and Respiratory Disease in Adulthood: A Population-Based Case-Control Study. Am J Respir Crit Care Med 2009;180:176-80

3. Baraldi E, Filippone M. Chronic lung disease after premature birth. N Engl J Med 2007;357:1946-1955.

4. Narang I, Baraldi E, Silverman M, Bush A. Airway function measurements and the long-term follow-up of survivors of preterm birth with and without chronic lung disease. Pediatr Pulmonol 2006;41:497-508.

5. Kharitonov SA, Barnes PJ. Exhaled markers of pulmonary disease. Am J Respir Crit Care Med 2001;163:1693-1722.

6. Berry MA, Shaw DE, Green RH, Brightling CE, Wardlaw AJ, Pavord ID. The use of exhaled nitric oxide concentration to identify eosinophilic airway inflammation: an observational study in adults with asthma. Clin Exp Allergy 2005;35:1175-1179.

7. Baraldi E, Azzolin NM, Zanconato S, Dario C, Zacchello F. Corticosteroids decrease exhaled nitric oxide in children with acute asthma. $J$ Pediatr $1997 ; 131: 381-385$

8. Piacentini GL, Bodini A, Zerman L, Costella S, Zanolla L, Peroni DG, Boner AL. Relationship between exhaled air temperature and exhaled nitric oxide in childhood asthma. Eur Respir J $2002 ; 20: 108-111$.

9. Paredi P, Kharitonov SA, Barnes PJ. Faster rise of exhaled breath temperature in asthma: a novel marker of airway inflammation? Am J Respir Crit Care Med $2002 ; 165: 181-184$ 
10. Paredi P, Kharitonov SA, Barnes PJ. Correlation of exhaled breath temperature with bronchial blood flow in asthma. Respir Res 2005 ;6:15.

11. Pifferi M, Ragazzo V, Previti A, Pioggia G, Ferro M, Macchia P, Piacentini GL, Boner AL. Exhaled air temperature in asthmatic children: a mathematical evaluation. Pediatr Allergy Immunol 2009;20:164-171.

12. Global Strategy for Asthma Management and Prevention, Global Initiative for Asthma (GINA) 2008. Available from: http://www.ginasthma.org.

13. Piacentini GL, Peroni D, Crestani E, Zardini F, Bodini A, Costella S, Boner AL. Exhaled air temperature in asthma: methods and relationship with markers of disease. Clin Exp Allergy 2007;37:415-419

14. Baraldi E, de Jongste JC; European Respiratory Society; American Thoracic Society. Measurement of exhaled nitric oxide in children, 2001. Eur Respir J 2002;20:223-237.

15. Miller MR, Hankinson J, Brusasco V, et al; ATS/ERS Task Force. Standardisation of spirometry. Eur Respir J 2005 ; 26:319-338.

16. Vrijlandt EJ, Boezen HM, Gerritsen J, Stremmelaar EF, Duiverman EJ. Respiratory health in prematurely born preschool children with and without bronchopulmonary dysplasia. J Pediatr 2007;150:256-261.

17. Allen J, Zwerdling R, Ehrenkranz R, et al. Statement on the care of the child with chronic lung disease of infancy and childhood. Am J Respir Crit Care Med 2003;168:356-396

18. Bhandari A, Bhandari V. Pitfalls, problems, and progress in bronchopulmonary dysplasia. Pediatrics 2009;123:1562-1573

19. Baraldi E, Bonetto G, Zacchello F, Filippone M. Low exhaled nitric oxide in schoolage children with bronchopulmonary dysplasia and airflow limitation. Am J Respir Crit Care Med 2005;171:68-72. 
20. Piacentini GL, Bodini A, Peroni D, Ress M, Costella S, Boner AL. Exhaled air temperature and eosinophil airway inflammation in allergic asthmatic children. J Allergy Clin Immunol 2004;114:202-204.

21. Thébaud B, Abman SH. Bronchopulmonary dysplasia: where have all the vessels gone? Roles of angiogenic growth factors in chronic lung disease. Am J Respir Crit Care Med 2007;175:978-985.

22. Coalson JJ. Pathology of bronchopulmonary dysplasia. Semin Perinatol 2006;30:179-184.

23. Abman $\mathrm{SH}$. The dysmorphic pulmonary circulation in bronchopulmonary dysplasia: a growing story. Am J Respir Crit Care Med 2008;178:114-115

24. Filippone M, Sartor M, Zacchello F, Baraldi E. Flow limitation in infants with bronchopulmonary dysplasia and respiratory function at school age. Lancet 2003;361:753-754.

25. Tiddens HA, Hofhuis W, Casotti V, Hop WC, Hulsmann AR, de Jongste JC. Airway dimensions in bronchopulmonary dysplasia: implications for airflow obstruction. Pediatr Pulmonol 2008;43:1206-13.

26. Jacob SV, Coates AL, Lands LC, MacNeish CF, Riley SP, Hornby L, Outerbridge EW, Davis GM, Williams RL. Long-term pulmonary sequelae of severe bronchopulmonary dysplasia. J Pediatr 1998;133:193-200.

27. Gross SJ, lannuzzi DM, Kveselis DA, Anbar RD. Effect of preterm birth on pulmonary function at school age: a prospective controlled study. J Pediatr 1998;133:188-192.

Doyle LW, Faber B, Callanan C, Freezer N, Ford GW, Davis NM. Bronchopulmonary dysplasia in very low birth weight subjects and lung function in late adolescence. Pediatrics 2006;118:108-113. 
Fletcher $G$, Peto $R$. The natural history of chronic aifflow obstruction. Br Med $d$ 1977;1:1645-1648. 


\section{FIGURE LEGENDS}

Figure 1. Panel $A$ shows PLET values in the 3 groups; panel $B$ shows $F E_{N O}$ values in the 3 groups; panel C shows FVC values in the 3 groups; panel D shows FEV1 values in the 3 groups. ${ }^{* *} p<0.001$ and ${ }^{*} p<0.01$ vs healthy controls; $\# p<0.05$ and $\xi p<0.01$ vs asthmatic subjects $\square p<0.001$ vs BPD survivors

Figure 2. Correlation between $\mathrm{FE}_{\mathrm{NO}}$ values and PLET values in BPD, asthmatic and healthy subjects $(R=0.42 p=0.004)$. 


\begin{tabular}{|l|l|l|l|}
\hline & Healthy & Asthma & BPD \\
\hline Number (males) & $17(11)$ & $17(10)$ & $17(7)$ \\
\hline Age (y) & $14.4(1.8)$ & $13.3(2.1)$ & $14.5(2.0)$ \\
\hline Height (cm) & $163.6(7.5)$ & $159.5(9.1)$ & $161.5(11)$ \\
\hline Weight (Kg) & $56.5(9.4)$ & $57.9(12.9)$ & $53.9(12.2)$ \\
\hline FVC (\%pred) & $106[99-110]$ & $101[95-107]$ & $85[77-100]^{\star \#}$ \\
\hline FEV ${ }_{1}(\% p r e d)$ & $104[100-111]$ & $88[76-93]^{\star \star}$ & $66[58-82]^{\star \star \S}$ \\
\hline FEF $_{25-75}(\% p r e d)$ & $121[107-133]$ & $75[57-87]^{\star \star}$ & $46[40-67]^{\# \star *}$ \\
\hline FEV $_{1} /$ FVC & $91[88-93]$ & $78[76-84]^{\star \star}$ & $72[67-80]^{\star \star}$ \\
\hline
\end{tabular}

Table. Demographic characteristic and lung function measures of study subjects. Anthropometric data are expressed as mean (SD). Spirometric data are expressed as median and IQR. ${ }^{* *} p<0.001$ and ${ }^{*} p<0.01$ vs healthy controls; $\# p<0.05$ and $\$ p<0.01$ vs asthmatic subjects. 


\begin{abstract}
A.
\end{abstract}

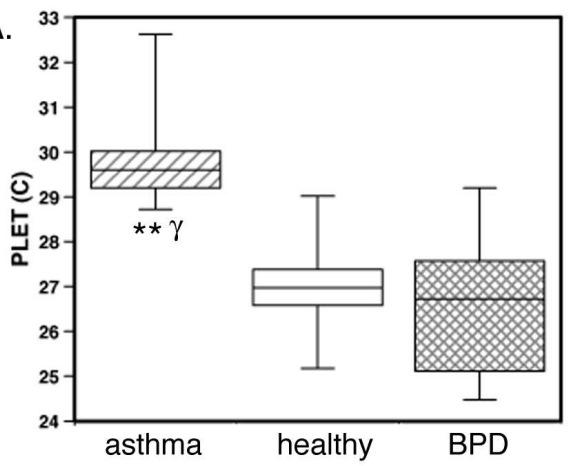

B.
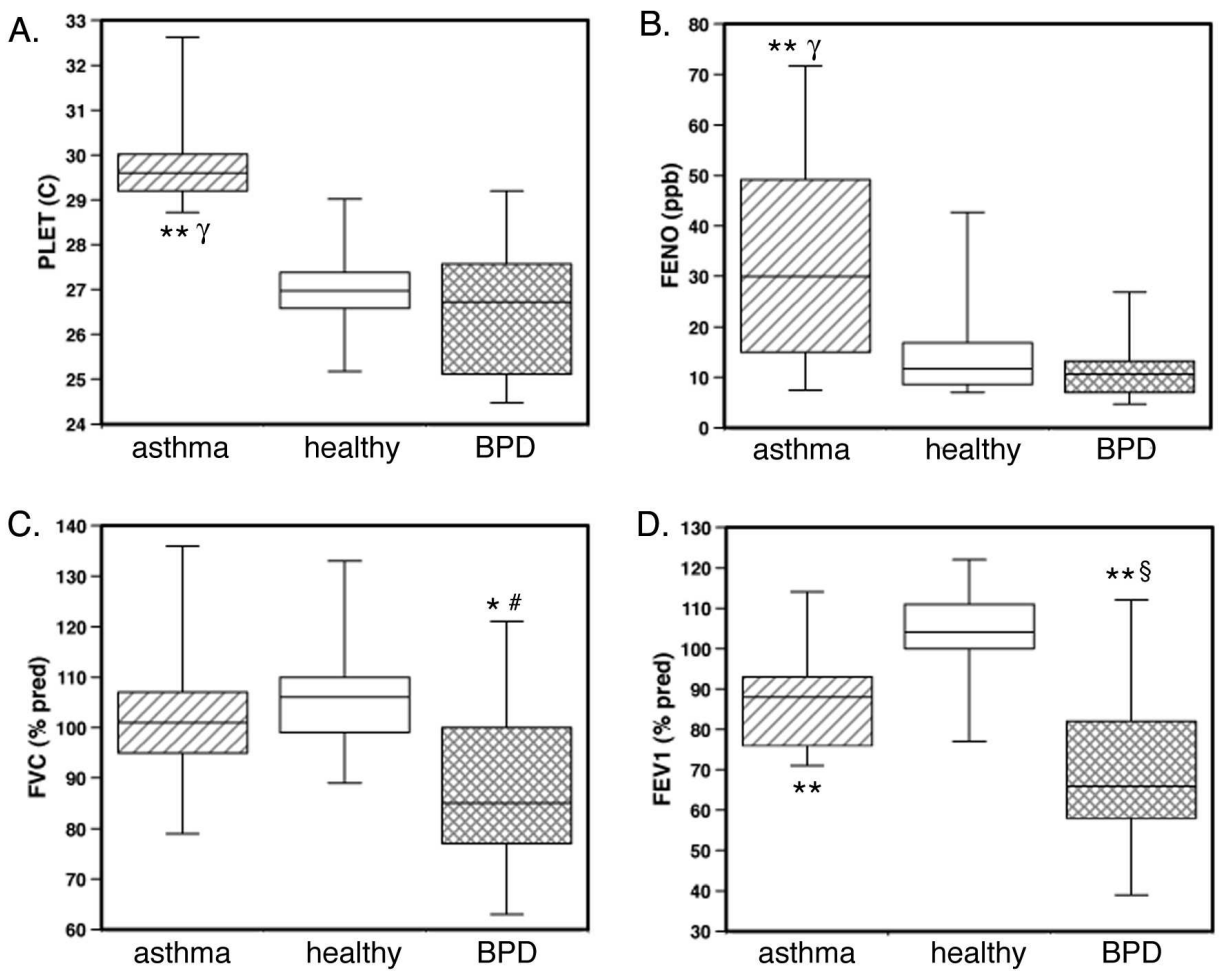

$160 \times 123 \mathrm{~mm}(300 \times 300 \mathrm{DPI})$

John Wiley \& Sons, Inc. 


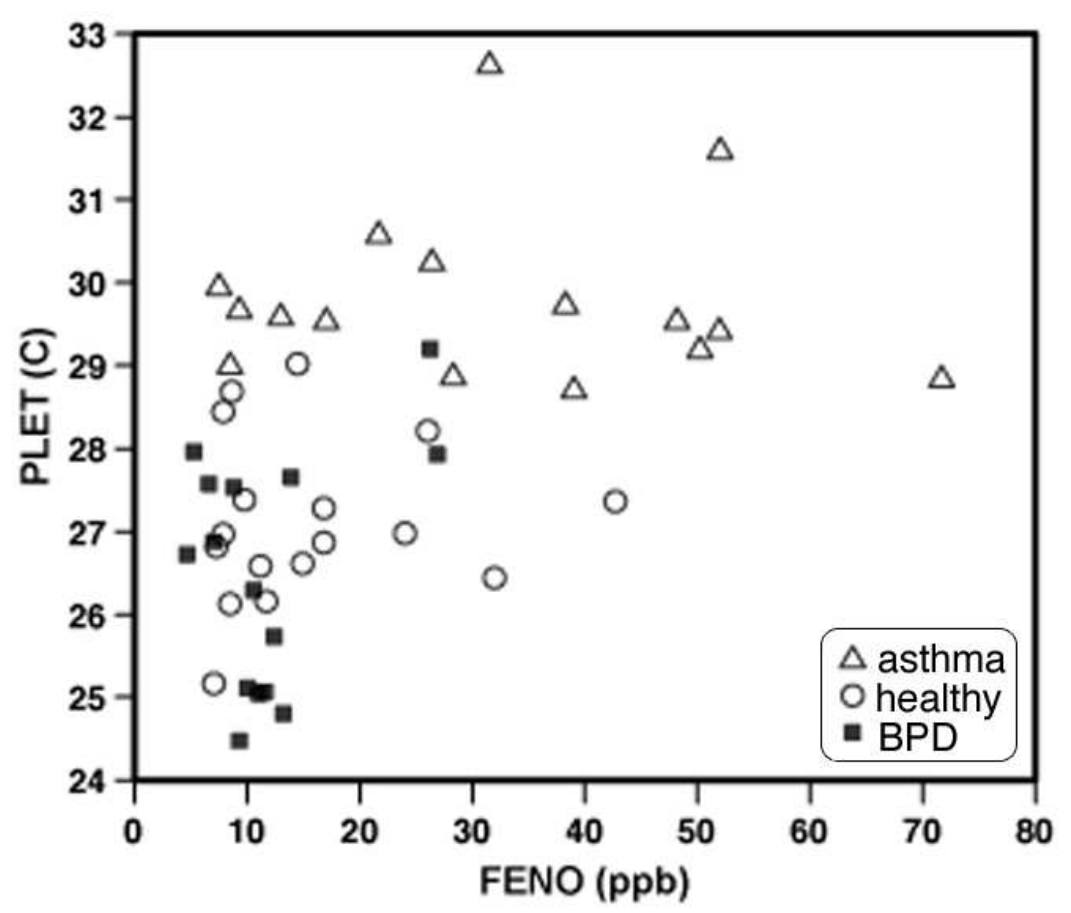

$70 \times 53 \mathrm{~mm}(300 \times 300 \mathrm{DPI})$

John Wiley \& Sons, Inc. 